\title{
Mechanistic Modeling Expedites the Development of Spray Dried Biologics
}

Carrigy, N.B. ${ }^{a}$; Liang, L. ${ }^{b}$; Wang, H.a ; Kariuki, S. ; Nagel T.E. ${ }^{\text {d }}$; Connerton, I.F. ${ }^{\text {; }}$; Vehring, R. ${ }^{\mathbf{a}^{*}}$

${ }^{\text {a }}$ Department of Mechanical Engineering. University of Alberta, Edmonton, Canada.

${ }^{b}$ School of Biosciences. University of Nottingham, Nottingham, UK.

${ }^{\mathrm{c}}$ Centre for Microbiology Research. Kenya Medical Research Institute, Nairobi, Kenya.

d Phages for Global Health. Oakland, USA.

*E-mail of the corresponding author: reinhard.vehring@,ualberta.ca

\begin{abstract}
Spray drying can be used to extend the shelf life of biologics stored at ambient temperature. Empirical and statistical design of experiments approaches typically require a relatively large number of experiments to determine suitable formulation and spray drying process parameters. An alternative approach, which may require fewer experiments, is to use mechanistic models to select these parameters. In this paper, mechanistic models are applied to develop a bacteriophage powder expected to have long-term physical stability at ambient temperature. The developed powder may be useful for decreasing incidences of foodborne illness in Kenya.
\end{abstract}

Keywords: bacteriophage powder; glass transition temperature; supplemented phase diagram; spray drying; stability. 


\section{Introduction}

Empirical and statistical design of experiments approaches are commonly used for selecting formulation and process parameters during the development of spray dried biologic products. An alternative approach is to use mechanistic models developed based on an understanding of fundamental underlying principles. That is the approach undertaken in this work wherein a dry powder bacteriophage (phage) dosage form is designed to have target characteristics without requiring a substantial number of spray drying experiments. The powder is intended for use in broiler chicken feed to decrease Campylobacter jejuni in the chicken gut, with the end goal of decreasing the prevalence of foodborne illness in Kenya, which is abnormally high. ${ }^{[1,2]}$ The development process can be divided into the following steps, discussed individually in this paper: 1) formulation; 2) atomization; 3) solvent evaporation and particle formation; 4) particle collection and analysis; 5) storage and transport.

\section{Materials and Methods}

A modified Büchi B-191 laboratory-scale spray dryer (Büchi Labortechnik AG; Flawil, Switzerland), schematically shown in Figure 1, was used to spray dry different myoviridae phages that can infect Campylobacter jejuni: CP30A, CP20, and CP8. These phages were isolated from chicken excreta and are present in chicken gut. ${ }^{[3]} \mathrm{A}$ transmission electron micrograph (TEM) of phage CP20 is given in Figure 2.

$Q_{f}=$ liquid feed flow rate

$\mathrm{Q}_{\mathrm{ag}}=$ atomizing gas flow rate

$\mathrm{Q}_{\mathrm{dg}}=$ drying gas flow rate

$T_{\text {in }}=$ inlet temperature

$\mathrm{T}_{\text {out }}=$ outlet temperature

$\mathrm{P}_{\mathrm{ag}}=$ atomizing gas pressure

$\mathrm{P}_{\text {out }}=$ outlet gauge pressure

$P_{\text {out }}-P_{c}=$ cyclone pressure drop

SPRAY DRYER
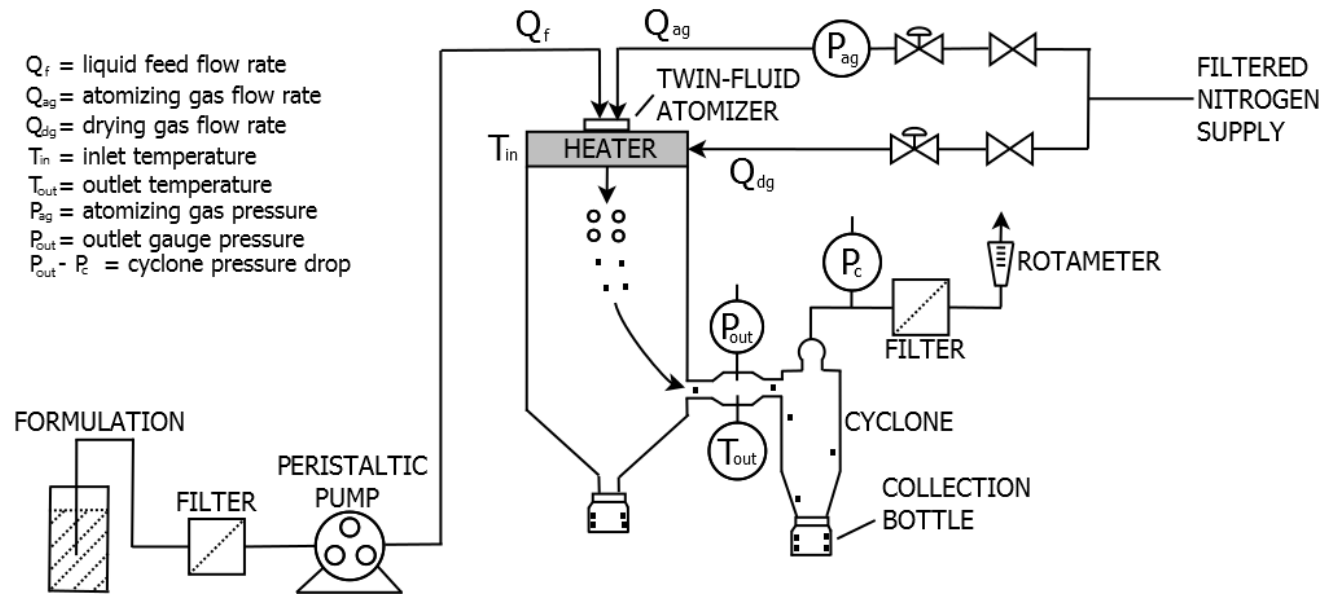

Fig. 1 Schematic of the modified laboratory-scale Büchi B-191 spray dryer. 


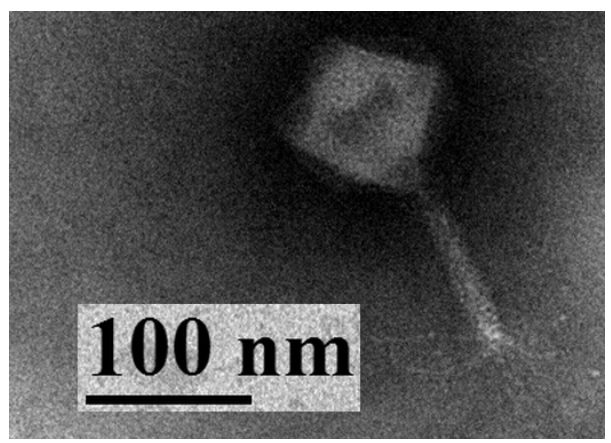

Fig. 2 TEM of phage CP20.

\subsection{Formulation}

The formulations were chosen to consist of L-leucine (Fisher Scientific, Cat. No. AC125121000; NH, USA) and D-(+)-trehalose dihydrate (Fisher BioReagents, Cat. No. BP2687; NH, USA) as dissolved solids in aqueous solution with phage ( $\mathrm{pH} \sim 6.5$ ). Trehalose is thought to stabilize biologics on drying by forming an amorphous glass that allows hydrogen bonds lost on desiccation of the biologic to be replaced. ${ }^{[4]}$ Leucine forms a shell that decreases particle cohesiveness. ${ }^{[5]}$

For spray drying experiments, stock phage lysates were filtered and centrifuged to reduce the impurity content to $<0.5 \mathrm{mg} \mathrm{mL}^{-1}$, and then diluted 1:100 into two aqueous formulations: (F1) $7.5 \mathrm{mg} \mathrm{mL}^{-1}$ leucine, $22.5 \mathrm{mg} \mathrm{mL}^{-1}$ trehalose; (F2) $12 \mathrm{mg} \mathrm{mL}^{-1}$ leucine, $18 \mathrm{mg} \mathrm{mL}^{-1}$ trehalose. In the literature similar formulations have been successfully used to stabilize spray dried phage. ${ }^{[6-9]}$ Further details regarding the choice of these formulations are given in the following sections. Plaque assay was used to measure the presence and extent of titer reduction due to dilution of phage into the above formulations. The results are presented in Section 3.1.

\subsection{Atomization}

The spray dryer uses a twin-fluid atomizer to generate droplets. An atomizing gas flow rate of $1.5 \times 10^{-4} \mathrm{~kg} \mathrm{~s}^{-1}$ and a spray rate of $1.7 \times 10^{-5} \mathrm{~kg} \mathrm{~s}^{-1}$ were used. A characteristic shear rate on the order of $1 \times 10^{5} \mathrm{~s}^{-1}$ was expected according to a model presented by Ghandi et al. ${ }^{[10]}$ In the literature, ${ }^{[7]}$ phage have remained active after atomization at similar shear rates. To verify minimal titer reduction, filtered phage CP30A lysate in formulation F1 was atomized onto a filter, from which liquid was drawn for assay. The results are presented in Section 3.2 .

The air-to-liquid ratio (ALR), defined as the ratio of the mass flow rates of atomizing gas and liquid feed, was 8.8. From the ALR and data for the present atomizer given by Hoe et al., ${ }^{[11]}$ an initial droplet diameter of $\sim 9 \mu \mathrm{m}$ was predicted. Using a model presented by 
Boraey et al., ${ }^{[12]}$ and the formulation compositions given in Section 2.1, the aerodynamic diameter at the time of shell formation was predicted to be $\sim 2 \mu \mathrm{m}$, which can be collected with the cyclone.

\subsection{Solvent Evaporation and Particle Formation}

Solvent from the liquid droplets generated by atomization quickly evaporates into the drying chamber of the spray dryer. The surface temperature of the droplets is typically assumed to remain near the wet bulb temperature for most of the evaporation process due to evaporative cooling ${ }^{[13]}$ therefore, thermal deactivation of the phage is not expected during initial stages of solvent evaporation. The dissolved solid that reaches critical supersaturation at the surface first may nucleate and form a crystalline shell. This is the particle formation stage, which has been described by Vehring et al. ${ }^{[14]}$ The formulation compositions in Section 2.1 were designed using mechanistic models such that leucine would reach supersaturation much earlier than trehalose, allowing for enough time to nucleate and crystallize leucine at the surface (11.5 milliseconds for F1 and 15.8 milliseconds for F2). Since previous work has demonstrated that conditions similar to F1 may not be sufficient for obtaining a fully crystalline leucine structure when phage lysate is present in the formulation, F2 was also tested with the expectation that it would form a fully crystalline leucine structure. ${ }^{[9]}$

A spray dryer process model, developed based on similar models in the literature, ${ }^{[15,16]}$ was used to predict the outlet temperature and the outlet relative humidity. The outlet temperature was predicted to be $\sim 50^{\circ} \mathrm{C}$ and the outlet relative humidity $\sim 3 \%$ for an inlet temperature of $70^{\circ} \mathrm{C}$, a spray rate of $1.7 \times 10^{-5} \mathrm{~kg} \mathrm{~s}^{-1}$, and a drying gas flow rate of $8.5 \times 10^{-3}$ $\mathrm{kg} \mathrm{s}^{-1}$.

\subsection{Particle Collection and Analysis}

A cyclone was used to collect the powder in a glass collection bottle. The collection efficiency is defined as the percent of the mass of dissolved solids in the feed solution recovered in the collection bottle. The collected powder containing phage was analyzed for solid state using Raman spectroscopy (using a custom device developed by Wang et al. ${ }^{[17]}$ ) and for particle morphology using scanning electron microscopy (SEM) (Zeiss Sigma FESEM, Oberkochen, Germany). Results are presented in Section 3.4.

\subsection{Storage and Transport}

Moisture uptake during storage and transport can result in crystallization of trehalose, which is known to deactivate phage. ${ }^{[18]}$ Therefore, it is crucial that the packaging material is moisture-equilibrated. In this study, a Steady State / Stability Test Chamber (910W-4, Lunaire Environmental, Williamsport, PA, USA) was used to equilibrate the powder and packaging to $4 \%$ relative humidity, with subsequent packaging occurring in the chamber. 
The relative humidity was chosen according to moisture uptake data and the information contained in Figure 3, described below. The packaging consisted of the powder in a vial, which was packaged in a double heat-sealed aluminum foil bag along with a satchel of silica gel desiccant (McMaster Carr, 2189K16; Elmhurst, USA), all of which were further packaged in another double heat-sealed aluminum foil bag along with another satchel of silica gel desiccant. The packaged spray dried powder, along with liquid controls, were shipped from Edmonton, Canada, to Nottingham, UK, in a Styrofoam box to minimize temperature variations. The titer after the complete development and shipping process will be determined by resuspension of the powder and plaque assay.

Throughout long-term stability studies, the powder should remain at a near-constant moisture content as, over time, moisture can lead to glass transition and crystallization. This tendency is demonstrated in a supplemented phase diagram that was developed for a trehalose-water system and is shown in Figure 3. With this diagram, the physical stability of the amorphous solid phase can be predicted a priori for a designed storage temperature, moisture content, and hence relative humidity. Use of this diagram is crucial for achieving long-term physical stability. The curve of the well-mixed glass transition temperature was developed using the Gordon-Taylor equation with a k-value of 5.9 and respective glass transition temperatures of $387 \mathrm{~K}$ for dry trehalose and $138 \mathrm{~K}$ for water. ${ }^{[19]}$ As a rule of thumb, long-term storage should be performed for conditions at least $50^{\circ} \mathrm{C}$ below the glass transition temperature. ${ }^{[20]}$

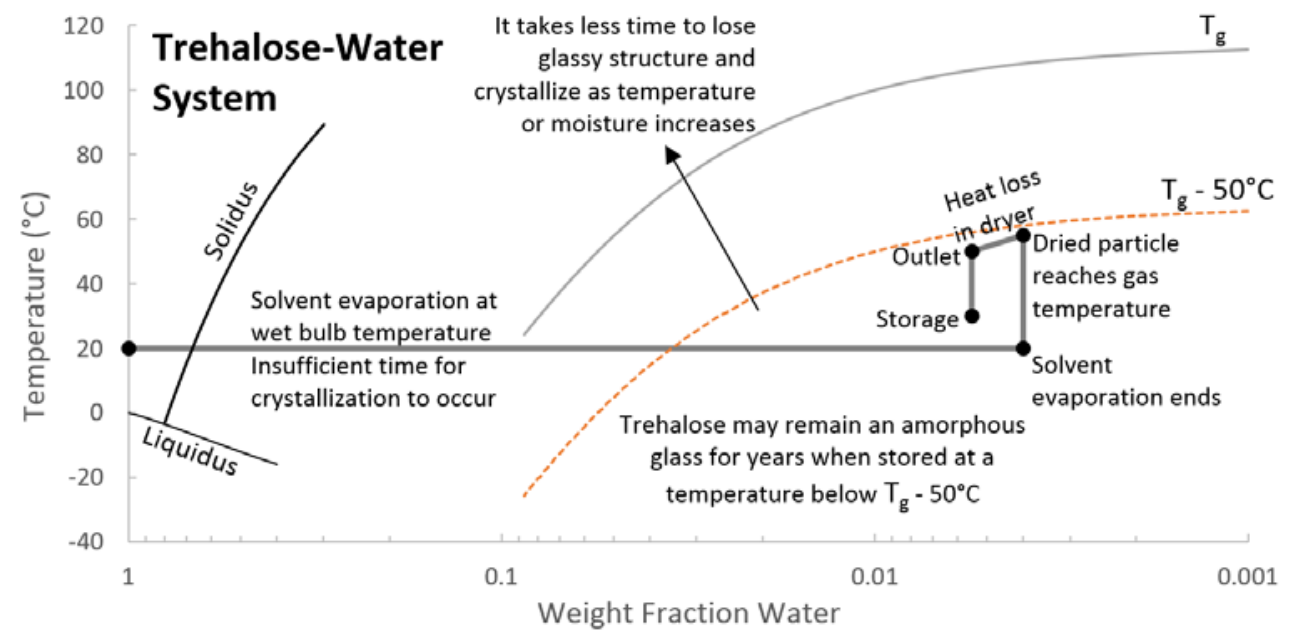

Fig. 3 Supplemented phase diagram developed for a trehalose-water system. Modified from Hoe et al. ${ }^{[21]}$ with the present process parameters and material properties. 


\section{Results and Discussion}

\subsection{Formulation}

Filtered phage $\mathrm{CP} 30 \mathrm{~A}$ lysate had no titer reduction over a period of 20 days when stored at $30^{\circ} \mathrm{C}$ in water, buffer, or formulation $\mathrm{F} 1$. A storage temperature of $40^{\circ} \mathrm{C}$ led to no titer reduction in buffer after 1 day but to $>1 \log (\mathrm{pfu} / \mathrm{mL})$ titer reduction after 10 days. A storage temperature of $50^{\circ} \mathrm{C}$ led to no titer reduction in buffer for at least 6 hours. No titer reduction was observed shipping $\mathrm{CP} 30 \mathrm{~A}$ in $\mathrm{F} 1$ within a vial in a Styrofoam box without temperature control from Nottingham, UK, to Edmonton, Canada, and back. This stability indicated that it is feasible to perform liquid control measurements with each experiment.

\subsection{Atomization}

The atomization titer reduction of phage $\mathrm{CP} 30 \mathrm{~A}$ in $\mathrm{F} 1$ was $\sim 0.25 \log (\mathrm{pfu} / \mathrm{mL})$, which is less than the value of $\sim 0.75 \log (\mathrm{pfu} / \mathrm{mL})$ reported in the literature for phages PEV2 and PEV40, ${ }^{[7]}$ where similar predicted shear rates were used.

\subsection{Solvent Evaporation and Particle Formation}

The outlet temperature matched predictions from the process model within $2^{\circ} \mathrm{C}$.

\subsection{Particle Collection and Analysis}

The collection efficiency was $54 \%$ for both formulations, which is typical for nominal batch sizes of $\sim 1.5$ grams. Raman spectroscopy confirmed trehalose was amorphous while leucine was mostly crystalline for both formulations. SEM (Figure 4) indicated that the phage lysate affected the particle morphology. It is possible that residual components in the phage lysate concentrated on the surface and interfered with the sensitive shell deformation and crystallization processes. This morphology was also present for other phage spray dried with leucine and trehalose in the literature, for which good powder flowability and longterm biological stability at $20^{\circ} \mathrm{C}$ were still achieved. ${ }^{[9]}$
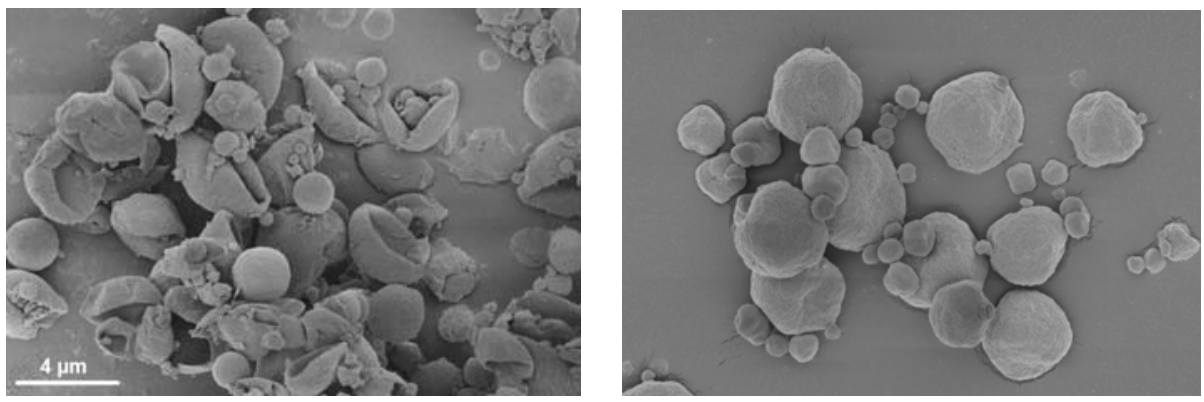

Fig. 4 SEM of spray dried F1 without (left) and with (right) phage CP20. The same scale bar applies to both images. 
Carrigy, N.B.; Liang, L.; Wang, H.; Kariuki, S.; Nagel, T.E.; Connerton, I.F.; Vehring, R.

\subsection{Storage and Transport}

The supplemented phase diagram in Figure 3 suggests that the developed powder will have long-term physical stability without the need for refrigeration when a low relative humidity is maintained in the packaging. The biological stability of the phage in the powder during storage and transport will be determined.

\section{Conclusions}

Mechanistic models were used to select formulation and spray drying process parameters for stabilizing phage. Further results will be presented at the conference.

\section{References}

[1] O’Reilly, C.E.; Jaron, P.; Ochieng, B.; Nyaguara, A.; Tate, J.E.; Parsons, M.B.; Bopp, C.A.; Williams, K.A.; Vinjé, J.; Blanton, E.; Wannemuehler, K.A.; Vulule, J.; Laserson, K.F.; Breiman, R.F.; Feikin, D.R.; Widdowson, M.A.; Mintz, E. Risk factors for death among children less than 5 years old hospitalized with diarrhea in rural western Kenya, 2005-2007: a cohert study. PLoS Medicine 2012, 9 (7), e1001256.

[2] World Health Organization, Food and Agricultural Organization of the United Nations \& World Organisation for Animal Health. The global view of campylobacteriosis: report of an expert consultation, Ultrecht, Netherlands, 9-11 July 2012. World Health Organization. http://www.who.int/iris/handle/10665/80751.

[3] Loc Carrillo, C.; Atterbury, R.J.; El-Shibiny, A..; Connerton, P.L.; Dillon, E.; Scott, A.; Connerton, I.F. Bacteriophage therapy to reduce Campylobacter jejuni colonization of broiler chickens. Applied and Environmental Microbiology 2005, 71 (11), 6554-6563.

[4] Crowe, J.H.; Carpenter, J.F.; Crowe, L.M. The role of vitrification in anhydrobiosis. Annual Review of Physiology 1998, 60, 73-103.

[5] Feng, A.L.; Boraey, M.A.; Gwin, M.A.; Finlay, P.R.; Kuehl, P.J.; Vehring, R. Mechanistic models facilitate efficient development of leucine containing microparticles for pulmonary drug delivery. International Journal of Pharmaceutics 2011, 409 (1-2), 156-163.

[6] Matinkhoo, S.; Lynch, K.H.; Dennis, J.J.; Finlay, W.H.; Vehring, R. Spray-dried respirable powders containing bacteriophage for the treatment of pulmonary infections. Journal of Pharmaceutical Sciences 2011, 100 (12), 5197-5205.

[7] Leung, S.S.Y.; Parumasivam, T.; Gao, F.G.; Carrigy, N.B.; Vehring, R.; Finlay, W.H.; Morales, S.; Britton, W.J.; Kutter, E.; Chan, H.K. Production of inhalation phage powders using spray freeze drying and spray drying techniques for treatment of respiratory infections. Pharmaceutical Research 2016, 33 (6), 1486-1496.

[8] Leung, S.S.Y.; Parumasivam, T.; Gao, F.G.; Carter, E.A.; Carrigy, N.B.; Vehring, R.; Finlay, W.H.; Morales, S.; Britton, W.J.; Kutter, E.; Chan, H.K. Effect of storage conditions on the stability of spray dried, inhalable bacteriophage powders. International Journal of Pharmaceutics. 2017, 521 (1-2), 141-149. 
[9] Leung, S.S.Y.; Parumasivam, T.; Nguyen, A.; Gengenbach, T.; Carter, E.A.; Carrigy, N.B.; Wang, H.; Vehring, R.; Finlay, W.H.; Morales, S.; Britton, W.J.; Kutter, E.; Chan, H.K. Effect of storage temperature on the stability of spray dried bacteriophage powders. European Journal of Pharmaceutics and Biopharmaceutics. 2018, 127, 213222.

[10] Ghandi, A.; Powell, I.B.; Howes, T.; Chen, X.D.; Adhikari, B. Effect of shear rate and oxygen stresses on the survival of Lactococcus lactis during the atomization and drying stages of spray drying: A laboratory and pilot scale study. Journal of Food Engineering 2012, 113 (2), 194-200.

[11] Hoe, S.; Ivey, J.W.; Boraey, M.A.; Shamsaddini-Shahrbabek, A.; Javaheri, E.; Matinkhoo, S.; Finlay, W.H.; Vehring, R. Use of a fundamental approach to spraydrying formulation design to facilitate the development of multi-component dry powder aerosols for respiratory drug delivery. Pharmaceutical Research. 2014, 31 (2), 449-465.

[12] Boraey, M.A.; Vehring, R. Diffusion controlled formation of microparticles. Journal of Aerosol Science. 2014, 67, 131-143.

[13] Masters, K. Spray Drying: An Introduction to Principles, Operational Practice and Applications; Leonard Hill: London, 1972.

[14] Vehring, R.; Foss, W.R.; Lechuga-Ballesteros, D. Particle formation in spray drying. Journal of Aerosol Science 2007, 38 (7), 728-746.

[15] Dobry, D.E.; Settell, D.M.; Baumann, J.M.; Ray, R.J.; Graham, L.J.; Beyerinck, R.A. A model-based methodology for spray-drying process development. Journal of Pharmaceutical Innovation 2009, 4 (3), 133-142.

[16] Ivey, J.W.; Vehring, R. The use of modeling in spray drying of emulsions and suspensions accelerates formulation and process development. Computers \& Chemical Engineering 2010, 34 (7), 1036-1040.

[17] Wang, H.; Boraey, M.A.; Williams, L.; Lechuga-Ballesteros, D.; Vehring, R. Lowfrequency shift dispersive Raman spectroscopy for the analysis of respirable dosage forms. International Journal of Pharmaceutics. 2014, 469 (1), 197-205.

[18] Vandenheuvel, D.; Meeus, J.; Lavigne, R.; Van den Mooter, G. Instability of bacteriophages in spray-dried trehalose powders is caused by crystallization of the matrix. International Journal of Pharmaceutics 2014, 472 (1-2), 202-205.

[19] Chen, T.; Fowler, A.; Toner, M. Literature review: supplemented phase diagram of the trehalose-water binary mixture. Cryobiology 2000, 40 (3), 277-282.

[20] Hancock, B.C.; Shamblin, S.L.; Zografi, G. Molecular mobility of amorphous pharmaceutical solids below their glass transition temperatures. Pharmaceutical Research 1995, 12 (6), 799-806.

[21] Hoe, S.; Boraey, M.A.; Ivey, J.W.; Finlay, W.H.; Vehring, R. Manufacturing and device options for the delivery of biotherapeutics. Journal of Aerosol Medicine and Pulmonary Drug Delivery 2014, 27 (5), 315-328. 